\title{
From America: Cookbook medicine or food for thought: practice guidelines development in the USA
}

\author{
Matthew H Liang
}

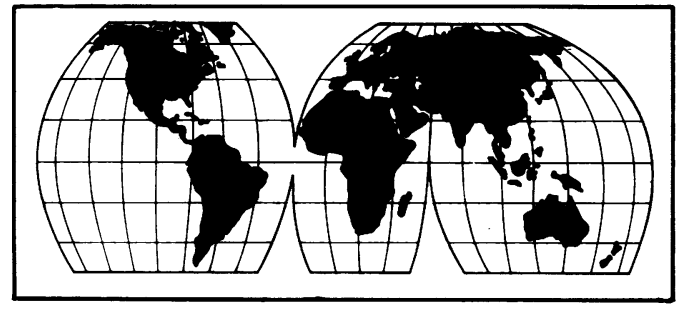

Practice guidelines have taken medicine in the USA by storm; over 1100 guidelines have been developed on subjects ranging from the management of carpal tunnel syndrome to urinary incontinence. Practice guidelines in one form or another have been around since the 1960 s when protocols or algorithms on common clinical problems were developed for health care workers who were not doctors, doctor's assistants, nurse practitioners, and health workers in a variety of settings in the USA and the Third World. Whether they are called practice policies, practice parameters, medical necessity guidelines, clinical indicators, review criteria, preferred practice patterns, or practice guidelines, they seek to provide explicit recommendations and to influence medical practice and decisions using a formal process to disseminate the best scientific evidence of effectiveness with expert's opinions. The lawyers prefer the term 'guidelines' because 'standards' implies inflexible rules and 'options' suggests vagueness and flexibility. Practice guidelines are either 'pathway' guidelines (similar to protocols or algorithms) which direct the doctor's management, or 'boundary' guidelines which define the limits of appropriate practice. Finally, guidelines are used to evaluate what doctors do versus what they should do and allow purchasers of care to identify value. ${ }^{1}$

The resurgence of interest in practice guidelines has been stimulated, in part, by research on the epidemiology of health resource utilisation. These studies show significant regional and international variations in surgical procedures and the variation is greatest in procedures with the most uncertainty regarding indications or outcome. Efforts to contain costs of care have focused on ways in which ineffective or unnecessary care can be identified and eliminated and tactics to inform doctors and their patients of these findings. With appropriate interventions, practice guidelines become standards of care. When acceptable alternative treatment exists, guidelines might define reasonable alternatives given the characteristics and preferences of the patient.

The American College of Rheumatology has been active but not more productive than other specialty societies in this effort. It has developed a guideline on the treatment of vague Lyme disease based on decision analysis and cost effectiveness analysis and is working on a guideline for asymptomatic hyperuricaemia and gout. Position statements have been written on bone density measurement, obtaining a rheumatology consultation, the rheumatologist's role in providing second opinions for reconstructive orthopaedic and neurological surgery, direction of physical and occupational therapy services for patients, arthroscopy by rheumatologists, and methotrexate. These have been based more on 'expert' opinion and consensus than on rigorous analysis.

Sixteen guidelines by the American Academy of Orthopaedic Surgery address such problems as carpal tunnel syndrome, total hip replacement, herniated lumbar disc, and osteoarthritis of the hip, but these are also general statements and not developed by formal review of existing data.

Practice guidelines from about 1991 are distinguished by a systematic attempt to summarise the existing information by quantitative synthesis (meta-analysis) and to use formal techniques to achieve explicit consensus by the nominal group or Delphi technique. ${ }^{2}$

Whether guidelines change behaviour, improve outcomes, or save money in North America is not clear. For instance, the guidelines recommending less use of caesarean section for breech births, patients who have had a previous caesarean section, and patients with dystocia were perceived by doctors as altering behaviour but the actual practice was not changed. ${ }^{3}$ Guidelines on cardiac pacemakers by the American Heart Association reduced the utilisation of pacemakers but the guidelines accompanied more restrictive reimbursement

The American Society of Anesthesiologists Guidelines for Monitoring general anaesthesia, however, prevented the occurrence of hypoxic brain damage and led to a reduction of malpractice premiums for anaesthesiologists. Guidelines for the management of patients receiving total hip arthroplasty in California led to a significant reduction in the average length of stay in hospital for the patient which was reflected in reduced costs, but this reduction
Harvard Medical School, Multipu

Department of Rheumatology/ Immunology, H Liang 
did not influence readmissions following surgery nor increased nursing home placements.

We read with interest that the experience with guidelines in the United Kingdom, at least in early studies, may be different. For instance, four different ways of disseminating guidelines from the Royal College of Radiologists on the use of chest radiography before an operation were compared with a control hospital. ${ }^{5}$ The best results were obtained by the appointment of a utilisation review committee which displayed the guidelines in surgical wards. Feedback on use of radiographs to consultants also produced a consistent reduction. The introduction of a new chest radiograph request form and concurrent review of requests by a radiologist had only a moderate effect.

The introduction of guidelines for the use of skull radiographs in patients with head injuries in the United Kingdom decreased utilisation, from $65 / 100$ attendances to $32 / 1000$. This was achieved without any untoward incidents and suggested a potential saving of 3.3 million pounds. $^{6}$

There is little evidence, but also little study, in America that any of the current practice guidelines have had wide circulation or acceptance or that most doctors know them. Doctors here and probably in the United Kingdom have traditionally been resistant to reductionist approaches to the practice of medicine. Part of this stems from experience and to the values of tailoring treatment to the needs and circumstances of an individual patient. Guidelines treat conditions as if they exist in isolation without any regard to whether the patient has multiple problems. The degree to which practice guidelines are viewed by doctors as being administrative fiats is another barrier to their acceptance.

Like many educational efforts, information is disseminated the most poorly to the groups at most need of the information. Specialists in musculoskeletal medicine and arthritis can hardly be helped by guidelines for rheumatic disease management but the inexperienced primary care doctor at the point of first contact does not have the time to read guidelines nor access to them. Social, rather than scientific, forces play the most important part in the evolution of practice and may explain why guidelines may be more effective in the United Kingdom. ${ }^{7}$ American doctors losing more and more autonomy are resigned to participating in this practice guideline exercise. To this observer, practice guidelines are unlikely to change practice themselves but the process of their development is more important than the outcome. Developing practice guidelines, like any explicit discussion of a difficult problem, can illuminate the areas of ignorance and identify the critical data necessary for decision making.

1 Eddy D M. Practice policies-what are they? FAMA 1990; 263: 877-80.

2 Field M J, Lohr $\mathrm{K} \mathrm{N}$, eds. Clinical practice guidelines: directions for a new program. Washington: National Academy Press, 1990: 1-160.

3 Lomas J, Anderson G M, Domnick-Pierre K, Vayda E, Enkin M W, Hannah W J. Do practice guidelines guide practice-the effect of consensus statement on the practice of physicians. N Engl Y Med 1989; 321: 1306-11.

4 Somers L S, Schurman D J, Jamison J Q, Woolson S T, Robison B L, Silverman J F. Clinician-directed hospital cost management for total hip arthroplasty patients. Clin Orthop 1990; 258: 168-75.

5 Fowkes F G R, Davies E R, Evans K T, et al. Multicenter trial of four strategies to reduce use of a radiological test. Lancet 1986; i: $367-70$.

6 Fowkes F G, Evans R C, Williams L A, Gehlbach S H, Cooke $B$ R, Roberts C J. Implementation of guidelines for the use of skull radiographs in patients with head injuries. Lancet 1984 ; ii: $795-6$.

7 Dixon A S. The evolution of clinical policies. Med Care 1990; 28: 201-20. 